\title{
Modeling VLE and LLE of Deep Eutectic Solvents (DES) and Ionic Liquids (IL) Using PC-SAFT Equation of State. Part II
}

\author{
Ali Aminian ${ }^{1}$ \\ ${ }^{1}$ Institute of Thermomechanics Czech Academy of Sciences
}

December 22, 2021

\begin{abstract}
This study aims to use Perturbed-Chain Statistical Associating Fluid Theory (PC-SAFT) to describe the phase behavior of systems containing DESs and ILs. The DESs are based on Tetrabutylammonium chloride $([\mathrm{N} 4444] \mathrm{Cl})$ and Tetrabutylammonium bromide (TBAB) as hydrogen bond acceptors, and levulinic acid (LevA) and Diethylene Glycol (DEG) as hydrogen bond donors in the mole ratio of 1:2 and 1:4, respectively. The predicted phase equilibrium data from PC-SAFT has been compared to those from COSMO-RS and NRTL predictions. ILs studied in this work are low viscosity ether-functionalized pyridiniumbased ILs [EnPy][NTf2] and [CmPy][NTf2], while 1-(2-methoxyethyl)-1-methylpyrrolidiniumbis(trifluoromethylsulfonyl)-amide) ([COC2mPYR][NTf2]) and 1-propyl-3-methylimidazolium bis\{trifluoromethylsulfonyl\}imide ([Pmim][NTf2]) were used for the study of the LLE systems with n-heptane + thiophene and n-hexane + ethylbenzene, respectively. In the last part, mixtures of linear alkanes and perfluoroalkanes have been studied to predict the phase behavior of perfluoroalkylalkanes with their linear alkane counterparts and comparisons have been made against SAFT-Mie pair potential.
\end{abstract}

\section{Hosted file}

AICHE_main2.pdf available at https://authorea.com/users/452340/articles/550440-modeling-vleand-lle-of-deep-eutectic-solvents-des-and-ionic-liquids-il-using-pc-saft-equation-ofstate-part-ii 